\title{
THEORETICAL FRAMEWORK OF SYSTEMS DESIGN FOR THE AIR TRANSPORTATION SYSTEM INCLUDING AN INHERENTLY QUANTITATIVE PHILOSOPHY OF SCENARIO DEVELOPMENT
}

\author{
Robin Ghosh*, Thomas Schilling*, Kai Wicke* \\ *German Aerospace Center (DLR)
}

\begin{abstract}
Keywords: air transportation system, systems analysis, scenario development, technology evaluation, requirements derivation, concept design
\end{abstract}

\begin{abstract}
This paper provides an overview of an inherently quantitative scenario philosophy for systems analysis and innovative concept design in the context of the Air Transportation System (ATS). A general perspective of the ATS is visualized in an "atomic model" with surrounding external scenario factors and the aircraft as the key connecting element between the main stakeholders: manufacturers, airlines, air navigation service providers (ANSPS) and airports. An iterative waterfall model is presented, which serves as a mental model of integration and decomposition over cascades of levels of detail from global scenario level to a single technology. The difference between classical scenario technique and a quantitative, yet participatory methodology of developing scenarios for the ATS is described. In order to integrate and decompose over a large span of levels of details, concept design and synthesis is as important as analysis. Further, quantitative scenario development may be considered as the synthesis of a skillful manipulation of a model deck. Scenario Gaming can be a method to simulate the settlement on requirements of complex socio-technological systems with multiple stakeholders and conflicting perspectives under radically changing boundary conditions. Scenario thinking can be an innovative and explorative instrument of participatory futurology, if not reduced to a mere "input for a tool chain".
\end{abstract}

\section{General Introduction}

Previous research on scenario development in aviation science was mainly focused on qualitative scenario technique, quantitative technology assessment for preliminary aircraft design or attempted to link these two concepts in a sequential way as in Strohmayer [1] and Phleps [2]. The theoretical framework presented in this paper is meant to encompass an approach and a view on the ATS which enables us to integrate previously disjointed theories, approaches and partial solutions into one overarching theory. The theoretical framework for systems design including systems analysis of the ATS and concept design with an integrated approach to participatory futurology is fundamentally different from approaches that can be found in the works of Meussen and Becker [3][4] as well as Eelman [5]. As an instrument of choice for participatory futurology we use scenarios. Herman Kahn, as a founder of modern term scenario, defined scenarios as "hypothetical succession of events with the objective of drawing attention to causal relationships and working towards decisions" [6][7]. Classical scenario technique based on consistency matrices inherently lacks the quantification which is required by systems and aircraft designers. Quantification is also needed in order to conduct systems analysis for sociotechnological planning on ATS level. We present a way of building "inherently quantitative" scenarios for the ATS as whole. The goal is a systematic and consistent framework for the ATS which is sufficiently abstracted in order to model and organize (in the ideal case) every possible research question concerning the ATS. To think the ATS as a whole does not mean to think every detail at once, but to understand the main driving interrelationships between stakeholders and external scenario factors. Because every 
systems analysis is different, a flexible framework is needed. This can only be realized by an extreme form of abstraction. Previous approaches using scenario methods in preliminary aircraft design [1-5] were almost exclusively based on sequential thinking with a quasi-linear process. During our research we gained the insight that this way of thinking is problematic for various kinds of questions which could not be answered satisfactorily. It was not possible to integrate partial analyses, models and design studies in a plausible way into the big picture we actually wanted to produce. The framework presented in this paper is highly non-linear as well as iterative in order to deal with complexity. On the one hand, the complexity is resulting from the long-term scope of futurology with the related timeline dynamics of scenario parameters. On the other hand, the scenario methodology needs to cope with great mental leaps from one level of detail to another, i.e. from single technology to the entire socio-economic system of the world and vice versa.

\section{General view on the ATS}

The "atomic model" of the ATS (see Figure 1) is a visualization of the core ATS consisting of the set of manufacturers, airlines (or aircraft operators in general), airports, and air navigation service providers (ANSPs), namely the 4-stakeholder model as described in Weiss et al. [8]. Within each stakeholder group, greatly different aspects like sub-elements, processes and infrastructures are consolidated. The 4stakeholder-model is one perspective on the core ATS and ATS-specific internal interactions. Surrounding external scenario factors [9] whose changes may influence the structure of the ATS (e.g. how aircraft are designed) or who may be influenced by a change of the ATS are depicted orbiting the core ATS. In one single analysis, both ways need to be considered. For example, a change in politics and technology may lead to changes of the core ATS and thus, the change of the ATS influences the environment in a positive and/or negative way.
Two main ways of exploring interrelationships of future changes between ATS core and external scenario shell are conceivable:

1. Impacts of hypothetical alterations of the ATS on external scenario factors over time.

2. Impacts that hypothetical alterations or goals of external scenario factors will or should have on the ATS over time.

The aircraft may be understood as the key connecting element between stakeholders. But stakeholders may also interact independent of aircraft, for example if only airports and airlines discuss the evaluation of technologies that potentially enhance the service level at a hub. Thus, stakeholders can also be analyzed reclusively or in interaction with other stakeholders without the aircraft as the connecting element.

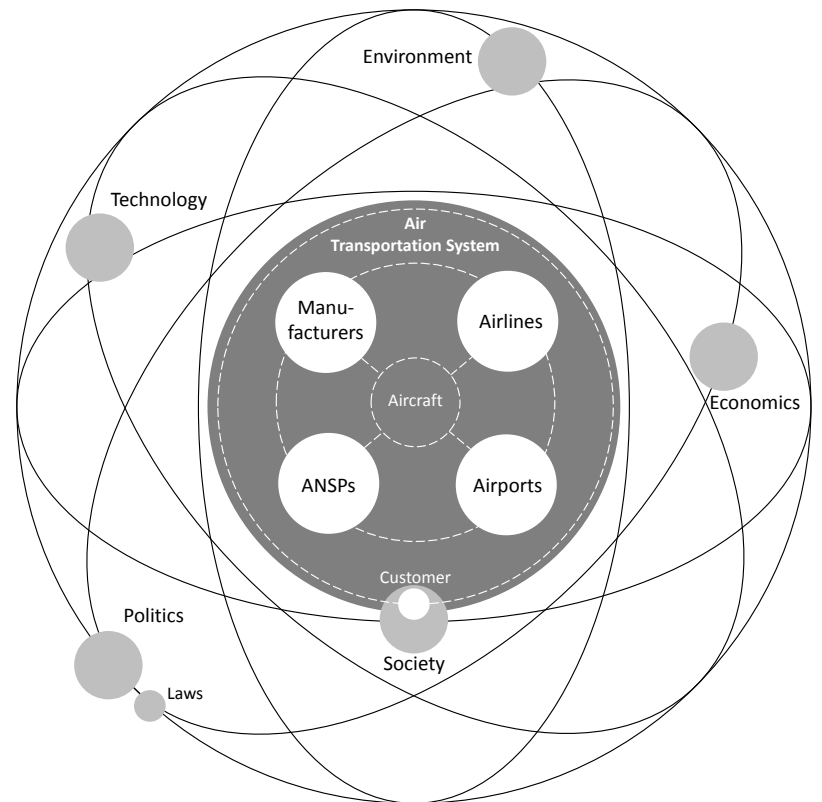

Figure 1: "Atomic model" with the four core stakeholders, the aircraft as the connecting element, and external key scenario factors including the customer

The aircraft manufacturer produces an aircraft with specific characteristics (flight performance, alternatively fueled, special maintainability, aircraft dimensions, passenger comfort, etc.). Future characteristics are settled upon with other stakeholders who impose constraints and requirements on the future aircraft from their 
perspective based on a given scenario. Note that in a future context (and to open the design space for innovative thinking), today's constraints and requirements may not apply in the future. In order to conduct innovative research, it may be even necessary to willingly ignore current constraints and requirements and "act as if" they did not exist. In order to design a useful scenario it may be also necessary adding new potential constraints to a static or dynamic scenario storyline ${ }^{1}$, even if it is not certain that they will be implemented. For example, we presuppose that a global climate target must be achieved or that peak-oil exists. We explore from that point in what way core stakeholders would be affected and how a holistic solution in such a scenario may look like. Under the postulated conditions, it then should be elaborated what key technologies at what performance figures would be needed to cope with the challenges of a scenario. Useful dynamic scenarios, which can be interpreted as chains of events, need a point of substantial change in their storyline, thus at least one key decision point. For the generation of decision scenarios it is favorable to act if one could change all parameters radically from a fictive omnipotent perspective. This includes the fictive implementation of global policies, the radical change of airport infrastructure as well as technological parameters (e.g. progress in battery technology enabling electric flight). Thus, it is helpful to act as the "principal engineer" or "architect" of the ATS, when in fact there is none, since it is de facto a selforganized complex system. This favors to elaborate the key decision points instead of relying on passive reactive analysis based on a forecast with an "incrementalist" mental model of the world. Following iterations of harmonizing between analysis and synthesis, goal setting and intermediate decisions, this will eventually end in the definition of requirements for e.g. aircraft design or airport concepts. This procedure will lead to a successive settlement on cost and performance requirements for single

\footnotetext{
${ }^{1}$ A definition of static and dynamic scenarios is given in Section 4.3.
}

key technologies to make those very concepts work.

In Figure 1 the customers are highlighted since transport demand is created by them. This can be customers of air freight or passengers. The customers are the reason why the ATS exists, but they are not deciding what kind of aircraft to buy or if the aircraft needs to fit into an infrastructure. This means that the customer is not involved in direct design or technology decisions of systemic relevance. The customer is indirectly involved in those decisions, but with no less importance to the ATS. Taking comfort as an example, passengers transfer their need for comfort through their choice to buy a ticket of a certain price, of a certain airline with a certain comfort proposition, but they will not negotiate with the manufacturer about seat pitch. Customer needs are indirectly connected through an interim stage over the airline or the airport to technology decisions. Our view of the society in context with the ATS is ambiguous, since two opposing positions can be taken. On the one hand, customers as a part of society create the demand and benefits of the value of air transport with sub-values like speed or frequency. On the other hand, as a part of society, customers may be harmed by the impact of noise or emissions of the system that they created the demand for in the first place. Therefore, they may want a constraint on cost of noise or emissions which thus has a dependent relationship upon speed or frequency, adverse to initial customer values.

Both the aircraft and the customer are considered as constituent elements of the ATS, the aircraft enabling the existence of the ATS from a technology side and the customer from a demand side.

\section{Iterative waterfall model - Technology, design, fleet and scenarios}

Key to the understanding of systems analysis with an inherently quantitative scenario philosophy for the ATS is the clear distinction of the scenario pull case (problem-deduced) and the technology push case (technology-induced). The iterative waterfall model (Figure 2) reflects these two idealized pathways. We distinguish 


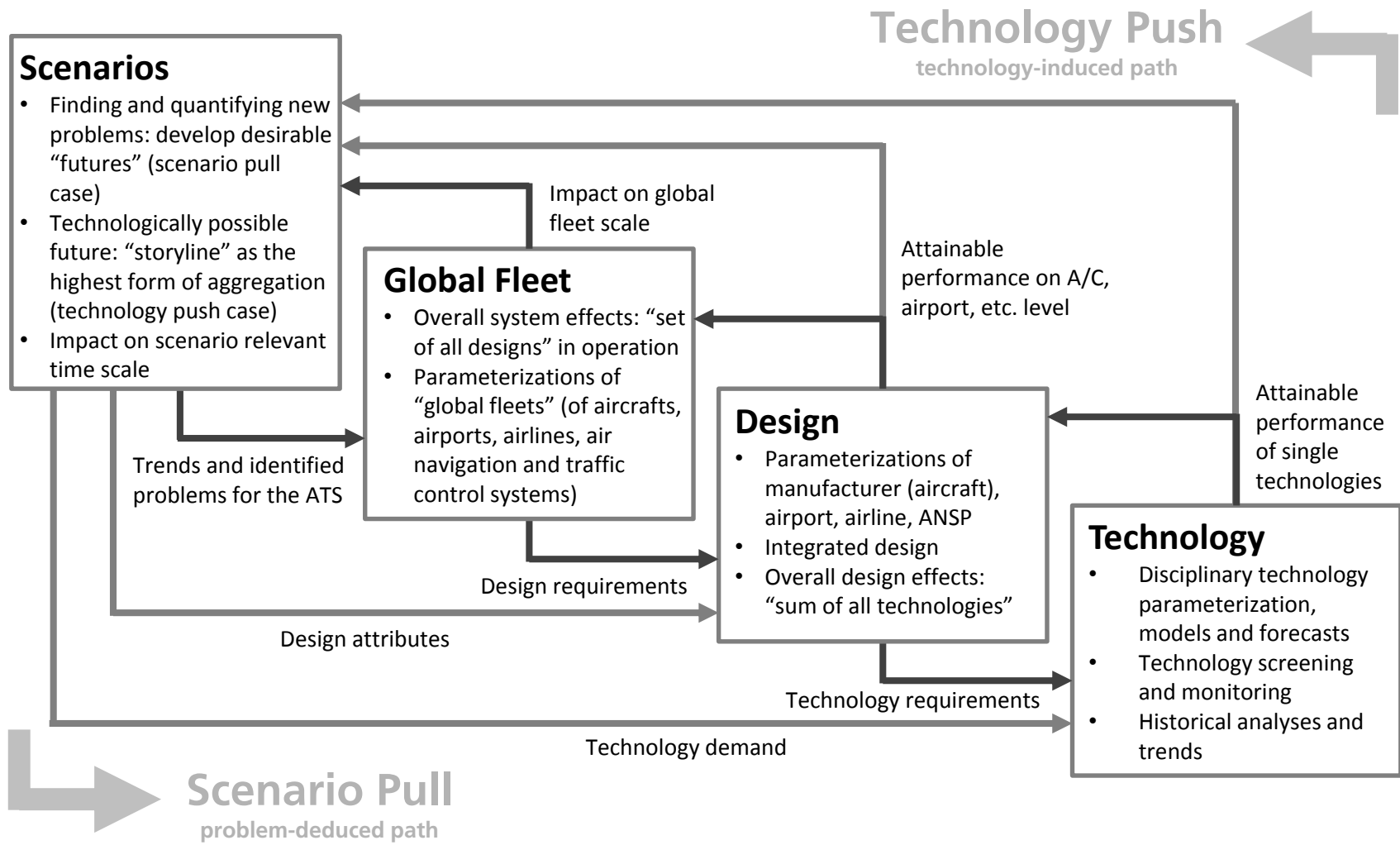

Figure 2: Iterative waterfall model with the four levels of detail scenarios, global fleet, design and technology with the according leaps to be taken from one level to another.

four levels of detail: Technology, design, fleet and scenarios. In context of the iterative waterfall model, "design" may be the conceptual design of aircraft as well as the design of airports, airlines or air traffic systems. The "global fleet" level as in Figure 2 is not necessarily just the fleet of worldwide aircraft, but also the "fleet of airports" or the "set of all airlines".

The clear distinction between pull and push cases is most important at the beginning of any process. In any of said cases, the order of elaborating a synthesis level by level before passing information on to the next higher or lower level is decisive. In this sense, the iterative waterfall model is also a mental model of integration and decomposition over cascades of levels of detail from global scenario level to a single technology. Analyzing and conceptualizing the ATS in a holistic way is especially difficult because of the variety of different levels of detail and associated leaps between levels. The iterative waterfall model reflects that systems analysis or scenario development concerning the overall ATS often has to overcome considerable logical leaps. They may be overcome stepwise with a welldefined narrative and mission formulation.

If it is the goal to deduce requirements for future aircraft, this would be a scenario pull case. An expectation of requirements derivation for future aircraft directly from an initial external scenario is unrealistic. This problem is mainly caused by skipping the fleet level since scenarios and aircraft design are not adjacent to one another in the iterative waterfall model.

The opposite direction of requirements derivation or scenario pull would be the technology push case. A disciplinary technology like e.g. laminar flow technology requires for evaluation first to be implemented in an aircraft design considering all design relevant trade-off and synthesis related effects. The attainable performances on single aircraft level are then analyzed on a fleet level, with operational aspects taken into account. These system effects or net benefit and cost can then be traded with relevant dynamically changing scenario factors 
(oil price, labor cost, environmental constraints, traffic growth) to support "go/no-go"-decisions for a certain technology.

At each level parameterization, sensitivity analyses and synthesis is required in order to build a scenario capability and link one level to the other. Starting a classical technology push case with a scenario analysis or using scenario technique very early in the process as suggested in [10] and [11] was unrewarding in the past since the evaluation process started simultaneously at two different ends of an analysis/synthesis process without the inbetween levels of design and fleet operation analysis/synthesis which are de facto the only means to link external scenario factors in a meaningful quantitative way to technology characteristics and performance. In the following, technology-induced as well as problem-deduced pathways are defined.

Technology-induced path: The design e.g. of an aircraft or an airport has to be conducted in order to assess the technology. The "fleet" level of detail has to be modeled in order to assess the design which requires modeling the operation of many aircraft or airports. In a last step, the fleet as well as identified relevant socio-economic conditions have to be brought on a timeline to evaluate the fleet level and develop decision scenarios. $^{2}$ An example is discussed in Section 10.

Problem-deduced path: At first explorative and normative scenarios are developed to find emerging problems and to estimate their extent and the urgency. Different scenarios are assessed and the desirable future is hypothetically selected from the set of scenarios. Based on this insight, the desired "fleet" evolution implies requirements on design level and on introduction timing of the "set of designs" enabling a desirable scenario. A design at a certain point in the future that delivers the characteristics and the performance required to satisfy the desired evolution at the fleet level implies requirements on characteristics and performance of a "set of technologies" at a

\footnotetext{
2 The term "decision scenario" refers to the reasoning of Pierre Wack in [12] [13].
}

certain time in the future that may in turn enable the required set of designs.

An example of a problem-deduced pathway is discussed in Section 9. It is of great importance to take the step by step approach and not to omit levels of detail, e.g. the fleet level between scenarios and design or the design level between fleet and technology. The information needs to be transported cascade by cascade to produce a consistent quantitative scenario logic.

The following section focusses on defining scope and purpose of a quantitative scenario philosophy for the ATS. We further discuss the main differences to classical scenario technique and the challenges that arise from the usage of the new methodology.

\section{An inherently quantitative scenario philosophy for the ATS}

Our main temporal scope of scenario development as an instrument of participatory futurology is strategic since we want to use insights for strategy development, strategy evaluation and assessment of go/no-go decisions. Strategic scenarios in this context refer to a scope of more than 20 years and to time steps between 1 and 10 years. Climate change issues for example may require an ultralong term structural assessment throughout the $21^{\text {st }}$ century as shown in [14]. Naturally, the ATS has a global spatial scope to which the scenario approach has to correspond. Therefore, the methodology is developed to produce global strategic scenarios.

\subsection{A Critique of scenario technique}

Scenario technique according to Gausemaier et al. [15] is not the instrument of choice to satisfy the demand of quantitative-oriented aeronautical future studies, since it is inherently qualitative. Classical scenario techniques published in aviation science are focused on the manufacturer or aircraft preliminary design alone. These techniques do not allow answering our research questions which do have a broader scope across different stakeholders. Classical scenario techniques using consistency matrices 


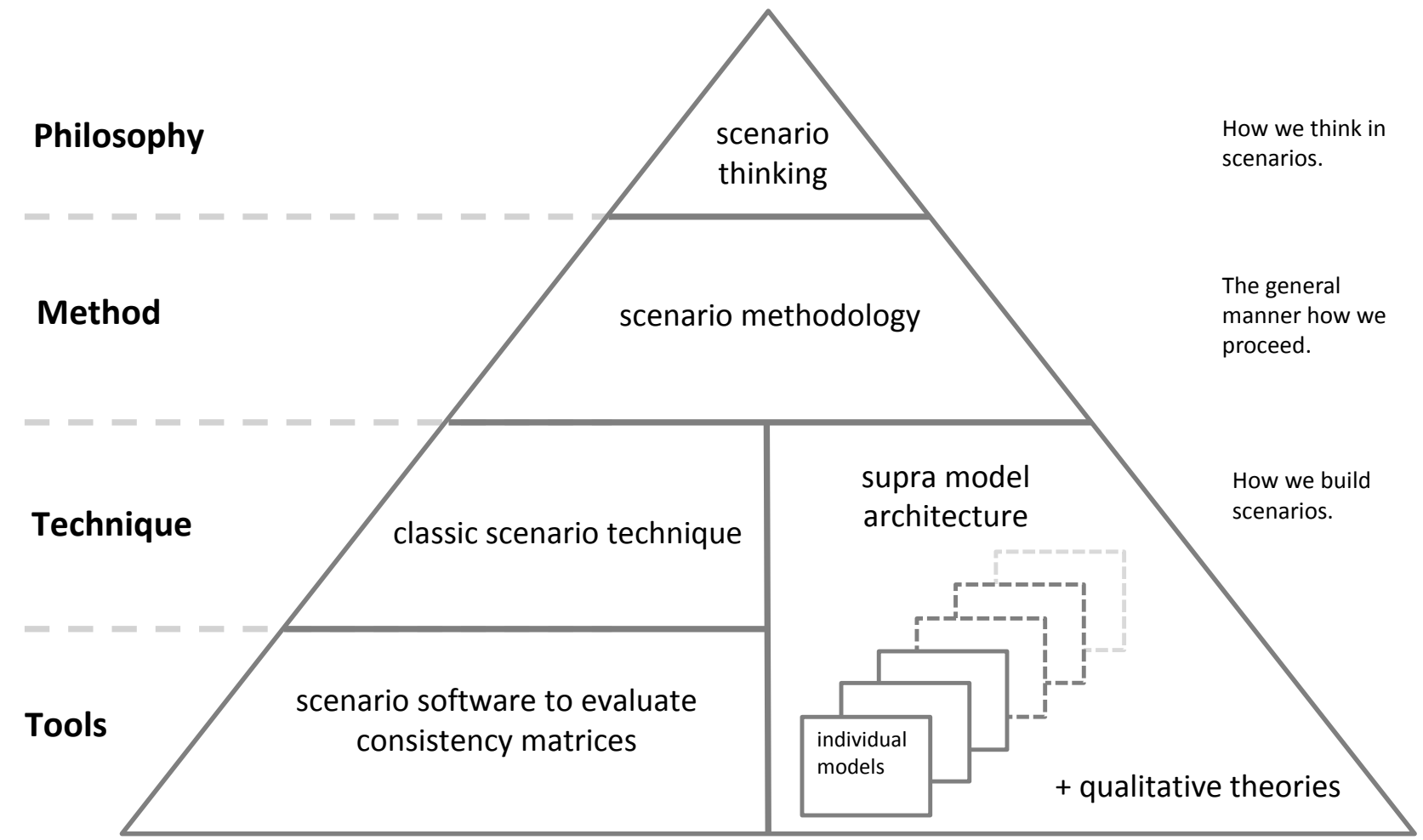

Figure 3: Inherently quantitative scenario philosophy (right) in contrast to prevalent scenario technique (left)

are for example able to produce a statement that two key factors might co-exist in a future, but not how they actually interrelate and by how much one parameter will change as a function of another one.

The main advantage of classical scenario technique is its generic character which can be applied rapidly and with low effort for very diverse scenario projects even across different problems and disciplines. Unfortunately, it inherently lacks quantification. As most of the times quantification is required for sound deduction of requirements for future aviation concepts, it is often tried to quantify the qualitative scenarios built with consistency matrices. Here, scenarios are developed first, with an attempt of post-scenario quantification. From this mental model results a widespread sequential and passive way of using scenarios that is neither adequate for coping with great complexity nor for developing non-incremental innovative concepts on ATS level.

As our key research object does not change (it is the ATS and will stay the ATS) and usually many scenario factors remain the same (with their development uncertain) ${ }^{3}$, it is acceptable to refrain from the benefits of the generic scenario technique and embrace the benefits of model-based scenario development instead. It is expected that the value of modeling the implication chain from one scenario factor throughout all levels of the iterative waterfall model is much higher for aeronautical research than trying to build qualitative scenarios with a mechanistic technique and quantifying the result in the aftermath.

\subsection{Scenario philosophy, method, technique and tools}

The main difference of the presented approach of scenario development in contrast to prevalent usage of a scenario technique oriented proceeding is depicted in Figure 3. While the

\footnotetext{
${ }^{3}$ A reappearing key scenario factor in most projects is for example the economic development described as gross domestic product (GDP). The development of the factor, how the GDP evolves over time (high, low, etc.), remains uncertain however.
} 
general philosophy of thinking in scenarios and the methodology are comparable, the decisive difference is found in the layers of used technique and tools. As a simplified analogy, the inherently quantitative scenario approach may be achieved by substituting technique ${ }^{4}$ and tool $^{5}$ by multiple numerical tools with an overarching architecture. The architecture defines how individual models or tools work together. The tools need to be manipulated by developers and experts of tools for this method. The required experience and skills to manipulate respective tools as a participant of quantitative scenario development is very high compared to classical scenario technique (see also section 7 and 8).

Classical scenario technique offers a step by step instruction for how to proceed and how to complete consistency matrices in workshops (where concerning the result, it does not matter if participants are experts or not). This step by step approach from identifying key scenario factors to developing different projections of those factors in order to check if they could coexist in a future is a technique (much like a recipe). A tool is defined as an entity that exists and can be used manually (e.g. a hammer or a software application). [16] In classical scenario technique the consistency matrices are usually evaluated by a scenario software tool. While the philosophy of thinking in scenarios and the general manner how we proceed are the same in both approaches, the technique is substituted by a supra model architecture that defines how individual models of sub-elements and subprocesses of the ATS are linked to one another. Numerical tools substitute the scenario software, where the main advantage of mathematical modelling is that we might discover how scenario factors and ATS parameters depend on one another in a quantitative way and not only that they could possibly co-exist. To ensure the effectiveness of scenarios when dealing with uncertainty, equal attention has to be paid to qualitative theories or possible radical changes even if not enough data

\footnotetext{
${ }^{4}$ chronologically prescribed proceeding

${ }^{5}$ implemented scenario software to evaluate consistency matrices
}

is available to decide on the probability of such changes or if the probability allegedly seems to be very low, while in fact it is uncertain ${ }^{6}$.

\subsection{Static and dynamic scenarios}

Generally, quantitative scenarios can differentiate between static and dynamic scenarios. In a static scenario, the story is not unfolding over a timeline, whereas in a dynamic scenario, the context as well as the research object change over time. In a dynamic scenario in contrast to a static one, elements and events of the scenario are put in a temporal relation, a chronological sequence, to one another. In a dynamic scenario it also matters when an event is taking place or which characteristic an element (or technology) has in a certain point in future time. In contrast, in a static scenario it only matters that an event occurs or an element of the scenario has a certain characteristic. In participatory futurology and quantitative scenario development in the context of the ATS, we are mainly interested in global scale and long term dynamic scenarios in which timing of events is considered.

In the technology push case, for example, static scenarios are usually built when potential parameter variations may yield too many possibilities which may reduce the contextual awareness instead of delivering clarification. In a next step, dynamic scenarios may communicate the meaning of the systems analysis in a time-relevant context.

\subsection{Qualitative and quantitative information in designing scenarios}

The great danger of an only-quantitative way of scenarios is that important issues of the future might be overlooked because there are no adequate metrics or no metrics have been defined yet. The most difficult problem for scenario development may be the merging of qualitative and quantitative information, which at first seems to be irreconcilable. Numerical models will not produce worthwhile scenarios

\footnotetext{
${ }^{6}$ Uncertainty is defined as the randomness of unknown probabilities. [7]
} 
without the creativity and ability of the operator to incorporate qualitative scenario information into his reasoning. This is fundamentally different to scenario technique and rather oriented towards a combination of model-based scenario development [7] and Peter Schwartz' "intuitive scenarios" [17].

It is of scientific value to actually test events (e.g. peak-oil, introduction of $\mathrm{CO} 2$ certificates, etc.) in the future with our model deck although we actually do not know if it actually will happen. It is more important to qualitatively understand the logic of peak-oil over the preoccupation with oil price scenarios. The scientific value of scenarios is not to predict a certain outcome, but to explore what consequences are implied if events will take place in a certain sequence. It might be difficult to convince facts-oriented people to hypothetically analyze or design under a scenario which is deemed "improbable", when in fact this would be an essential part of thinking in scenarios. In this context, scenarios can also help to generate innovation by opening minds and design spaces. This prevents aircraft design and systems design teams from producing more of the same. The design of aircraft for the future is based on the knowledge of the past and on an "incrementalist" mental model of the world. This may not be sufficient to cope with systemic and structural changes in the future. While thinking in scenarios, we refrain from ceteris paribus conditions, which is also a fundamental difference to the forecast. [16] In order to do this, "weak" qualitative information about the future has to be minded as much as quantitative information.

\subsection{Drawbacks of not using scenario technique}

The drawbacks of not using scenario technique but system models and developing scenarios with the latter are:

- No standardized process (risk of getting paralyzed by overwhelming complexity)

- Higher effort
- Not ad-hoc usable for questions for which no mathematical models exist (system models take time to develop)

- More complex (architecture required)

- Slower

- Shape of results less predictable (but therefore also potentially more innovative)

- Difficult to not automatically overemphasize quantitative over weak qualitative signals: constant need to remind the involved persons to not concentrate only on quantitative information

We estimate though, that the cost of development is justified by the quantification the methodology delivers with respect to potential evolutions of fleet and network, climate impact, policy impact, and technology decisions. This eventually leads to insights of a quality unachievable by scenario technique alone.

A method based on diverse mathematical models operated by individual experts developing a scenario with their tools in a defined setting may be rewarded with more relevance, quantitative scenarios and understanding of the driving interrelationships within the ATS and future key decision points. The complex nature of systems modeling might require to step back from the aspiration of creating consistent pictures including all aspects of the future at once and to start monocriterially, including more criteria stepwise.

Peter Schwartz states that "imaginative people with open minds that can work well together as a team" are an essential basis for creating useful scenarios. He further concludes that good scenarios are "plausible and surprising" and "have the power to break old stereotypes". [17] This is even more relevant in the inherently quantitative scenario approach based on a wide range of different mathematical models than in a predefined scenario technique process. Additionally, expert moderation for the process is essential. 


\section{Concept design vs. systems analysis}

In order to see the ATS as a whole it is not sufficient to focus on analysis only. Analyzing a system thoroughly cannot automatically lead to a fixed set of requirements for future aircraft. The settlement on requirements is only possible in a constant loop of analysis and synthesis. The deduction of requirements to the Design and Technology level as in Figure 2 will also be associated with a simultaneous conceptual design process. A necessary ingredient for requirements deduction is synthesis. Only analyzing an entity like the ATS based on today's data without creating new aspects and recomposing old ones will not provide sufficient insight to define requirements and cope with the challenges of the future. Conceptualizing means creating and converging on solutions under divergent drivers, while analysis focusses on assessing what is already there. Thus, in order to push requirements downward through the levels of the iterative waterfall model, it is necessary to conduct synthesis-oriented concept designs, even at ATS level. Thus, requirements deduction is as related to conceptual design and synthesis as it is to analysis. It is essential for the deduction of requirements that systems analysis and concept design alternate in a constant process, where systems analysis may discover and quantify future problems and the concept design may elaborate future solutions. Conducting thorough systems analysis does not only mean choosing the most promising alternative in terms of cost, time, effectiveness and feasibility, but also questioning the goals of the decision maker per se. [18] The goal of systems design including our scenario philosophy is not only to assess technologies but also to assess and develop new strategies and technology perspectives for future concepts in the field of aviation. Herman Kahn, while working at the RAND Corporation, already pointed out in the $1950 \mathrm{~s}$ that there are fundamental differences between analysis and

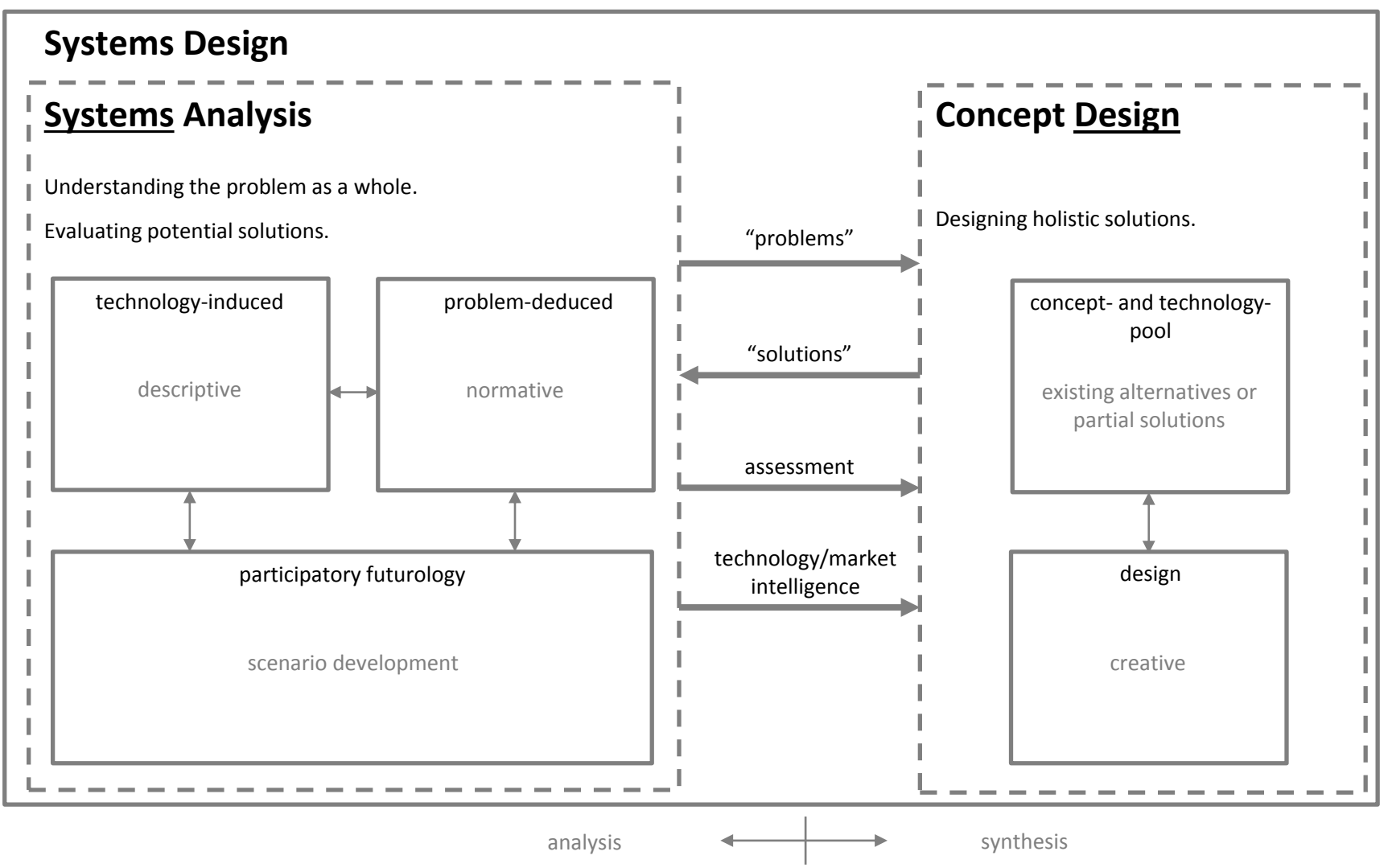

Figure 4: Systems design includes both analytical and conceptual aspects. While systems analysis defines problems, concept design synthesizes solutions which are then in turn analytically evaluated. Alternation between the opposed thinking concepts systems analysis and concept design is required to work towards innovative and holistic solutions. 
design. [19] In Figure 4, the distinction between systems analysis [20] and concept design is illustrated. Both parts require a different kind of thinking, one being rather analytical, the other being rather conceptual. Further, Kahn remarks that over-preoccupation with analytic details about what will happen or what to do after something unwanted has happened might restrain the analyst from asking the right questions. It is rather the question what can be done in advance that should lead the research. [19] Therefore, a distinction between systems analysis and design thinking has to be made and to be incorporated in a systems design theoretical framework. In order to deduce requirements for the ATS, it is favorable to think in a normative way instead of a descriptive one. It is rather the question "How should the future be?" than "How will the future be?" that leads to the derivation of useful requirements for a future system.

Knowing that design is at least as important as pure analysis for the deduction of requirements in a future system, the linkage between requirements derivation and scenario development is discussed in the following section.

\section{Scenarios, systems analysis, strategy and requirements for concept design}

Requirements for future aircraft do not directly result out of scenarios; in between, intermediate decisions have to be made and goals have to be set. The nexus between participatory futurology, systems analysis, strategy development and concept design is depicted in Figure 5. Requirements cannot be "calculated" directly from scenarios because the output of scenario analyses will still be uncertain and the number of possible alternative outcomes is infinite. Therefore, the derivation of requirements needs an early intermediate stage of decision making and goal setting against the backdrop of infinite possibilities. This is conducted by strategy development concerning a given question.

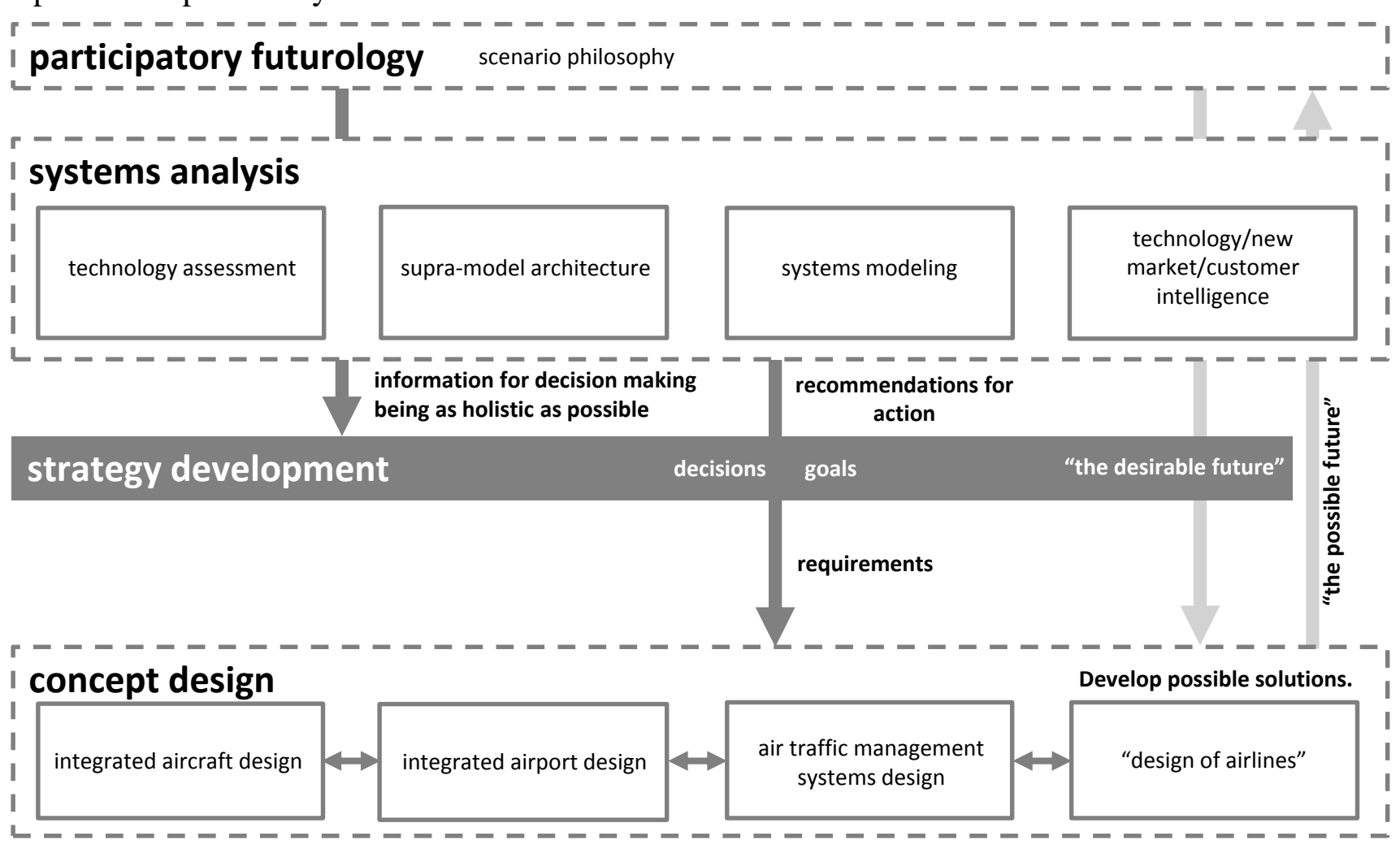

Figure 5: Interrelation of scenarios, systems analysis, strategy development and concept design. Requirements cannot be deduced without an interim stage of decision making and goal setting, reflecting and defining the "desirable future". 
Strategy development may be supported by future studies and holistic systems analysis. On the basis of a world view, a strategy and goals need to be developed and consequently, a "desirable future" must be formulated. From this point, requirements for the ATS and its stakeholders and sub-systems may be elaborated. On the other hand, a possible future is explored by concept design answering how and when a solution to meet future requirements on ATS level may be feasible.

As scenarios do not automatically result from systems models, requirements do not automatically result from scenarios. The only entity that can be altered by scenario development is the world view of decision makers. [12][13] These may also be engineers who make design decisions while conceptualizing, e.g., a new aircraft. Thus, before settling on requirements, intermediate strategic decisions have already to be made on the basis of scenario analyses.

In order to define intermediate goals and to investigate decisions during a participatory systems design process while considering perspectives of multiple stakeholders at once in a quantitative way under changing scenario conditions, Scenario Gaming, as described in Section 7, may be a valid method.

\section{Scenario Gaming - Where it can work}

Scenario Gaming integrates gaming aspects into quantitative scenario development. The first goal is to incorporate the tacit knowledge of experts and technologists as manipulators of numerical tools into the development of scenarios. The second goal is to simulate complex interactions between stakeholders under radically changing boundary conditions (see Figure 6). Scenario Gaming for ATS related future studies is derived from War Gaming, developed as a method of systems analysis as in [21]. The most important point of experimental planning games/simulations for scenario development and the overall design and evaluation process concerning the ATS is what Tsuchiya [22] calls the improvement of "the commensurability of interpretative frameworks" within a diverse project team with diverse knowledge. Especially in research projects with a long time horizon of more than 30 years, explicit as well as tacit knowledge of individual experts is essential for useful scenario development.

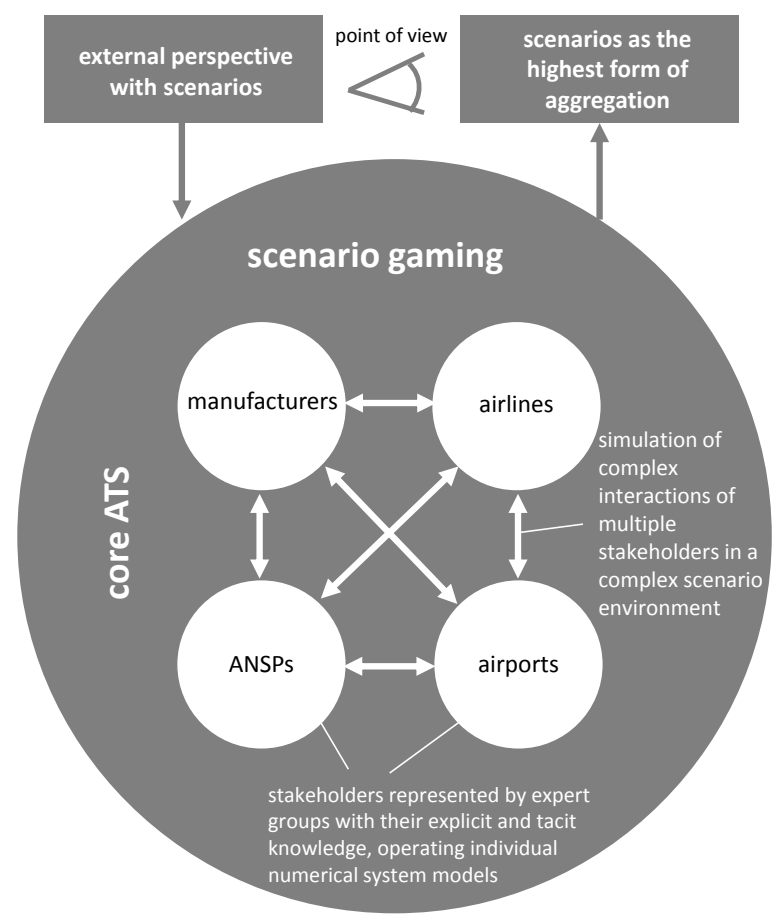

Figure 6: Simulation of interactions between conflicting priorities of stakeholders in a complex scenario environment

Tsuchiya further argues that games or simulations enable explicit and tacit knowledge of individuals to become organizational knowledge. Without commensurability of frameworks within an organization, "sharing personal knowledge to create organizational knowledge will not be possible." [22] Generally, while trying to elicit questions concerning the long-term future of aviation, the world view of participants of the scenario process might differ substantially. One effect might be that inferences do not converge into a set of consistent scenarios.

Joldersma and Geurts [23] emphasize that such games can push the participants to explicate their initial mental models, to reflect them and to react on feedback from other participants. Eventually the process will change the participants' mental model of the world and 
work towards a commensurable interpretative framework. This can also be interpreted as a part of the "internal synthesis" which constitutes a part of the overall scenario as described in Section 8.

As in Crookall et al. [24], gaming and simulation can help in three general areas. They formulate three archetypes of games which are here transferred to systems analysis and concept design questions of the ATS:

- "Knowledge-to-action": Use developed isolated design, assessment and optimization tools and personal knowledge and apply them integratively to a specific problem.

- "Action-to-knowledge": Use the work on a specific project to generate new knowledge and skills, build new features into design and assessment tools.

- "Integrating-action-knowledge": Game setting is designed to encourage participants to make connections between their tools and their related individual explicit and tacit knowledge across diverse disciplines. [24]

In practice, we use a combination of these archetypes at once. Scenario Gaming, as a scenario-oriented simulation game, can be a method to start a simulated interaction between the ATS's four main stakeholders in spite of the problem's vagueness or of a missing pathway towards a solution. It is a method to start a multi-disciplinary discussion about the future in a situation in which the interrelationships are unknown or fuzzy and where interfaces between disciplines are not yet existent or even contradictory; however, the wish is to define relationships and thus to develop real understanding of the driving forces. Scenario Gaming can work to overcome isolated problem-solving and to search for local optima (e.g. aircraft itself, airport itself, energy system itself, climate change itself) and island meditations with the idea of waiting for the right input for a developed tool.

Scenario Gaming helps to build a setting in which representatives of the main stakeholders [8] operating individual tools or partial tool chains can clarify a complex problem and interact with each other (see Figure 6). This is a viable option if linear processes for complex problems concerning the future of the ATS do not yield real progress or innovation. During the conflict simulation, essential decision points will be revealed. The synthesis should focus on these decision points in order to build decision scenarios as described by Pierre Wack in [12]. Insights gained from the simulation process over critical decision points need to be aggregated and incorporated into the scenarios to build the overall storyline.

It also is a method to elaborate target conflicts between stakeholders of the ATS represented by participants of the systems analysis and design teams and negotiate a variety of possible decisions between stakeholders.

Scenario Gaming may be a way to operate if the problem is opaque or difficult to define purely analytically at the beginning of a project. This is principally the case in aeronautical research projects about long-term future aviation concepts where past experience soon yields its limits. How will the world be in 2050 when e.g. a new technology or a new aircraft concept is introduced into the ATS? How should the aircraft look like to fit potential future requirements? In the context of futurology, these questions cannot be answered uniquely and conclusively. Yet, Scenario Gaming allows a step by step convergence to clearer formulation of problem and solution when confronted with divergent possibilities, without getting stuck in a linear process.

Scenario Gaming may be useful if fundamental changes of the system are expected or wanted (breakthrough innovation like zero emission aviation). This scope often means that similar cases do not exist so that past experience and tools need to be transferred into a completely new context.

Scenario Gaming is a people-oriented method like classical scenario technique. It however allows for simulating interactions between the main stakeholders of the ATS and multiple disciplines working with a deck of different individual numerical models (see also Figure 7). This raises the contextual awareness of the involved team and of the organization 
while at the same time giving more space for innovation and required synthesis.

By this means, scenarios are developed from not only for our quantitative models and numerical tools, but with them in equal measure. A collaborative environment like an Integrated Design Laboratory as described in [25] would be favorable, but creative and open-minded participants are essential. Model experts need to be competent in manipulating tools, in the overall understanding of the ATS, in working on a very abstract level and on different levels of detail in a multi-disciplinary team. Openmindedness and creativity is needed to overcome the initial incommensurability of frameworks and to cope with potential contradictory information in the design process. Collaborative Scenario Gaming is a promising approach to problems concerning the future of air transport that cannot exclusively be solved by analytical means. The idea of Scenario Gaming is generally compatible to the idea of concurrent engineering. Herman Kahn states that "rule games may be a useful way to summarize, integrate, and evaluate research that has been done in bits and pieces" and that "it also makes the lacunae stand out." [21]

In order to conduct such a participatory scenario process, steering moderation is crucial.

\section{Quantitative scenario development by model manipulation}

As already mentioned above, quantitative scenarios in context of the ATS might be built by skillful model manipulation. Scenarios are essentially organizing assumptions in terms of a communicable narrative. The presented theory relies on quantitative as much as on qualitative assumptions. For any quantitative scenario, additional qualitative assumptions complete a possible future scenario. Furthermore, surfacing "white gaps" between numerical models may need to be bridged with qualitative assumptions.

Usually, it is assumed that only external assumptions or "a plausible description of the future" (termed "input" or external assumptions in Figure 7) are "the scenario". In fact, it would be far more effective to consider input and output as the external scenario. Scenarios are not mere starting points for a linear chain of tool usage; in fact, they are likewise the result, "the story". This set of input assumptions and output results is one possible external scenario. If scenarios are considered only as a starting point where pictures of tomorrow are developed "out of the blue" without a precise objective, it is difficult if not impossible to develop quantitative decision scenarios. Not only external results should be viewed as part of the overall scenario but also the internal synthesis of the model deck and model specific internal assumptions. They are often hard-coded into some tools and equally often forgotten to be part of the overall scenario. Model specific internal assumptions could be altered resulting in interesting, innovative and surprising scenarios, because assumptions which are usually treated as fixed could and should be altered.

The total of a scenario is the set of all assumptions, whether external or internal, quantitative or qualitative. Another important component to the overall scenario is the internal synthesis of the model deck, here named the supra model architecture. In the architecture, the world view on the ATS and its internal interrelations are incorporated. This is based on assumptions on how the ATS and external scenario factors work and behave. With the instrument 'scenarios', the change of allegedly stable or unchangeable parameters can and should be tested.

In any case, scenarios should not be mistaken with forecasts. One of the most important tasks in the development process of scenarios is to work out critical decision points and to emphasize the implications of one decision over another. [12] In a scientific environment these are rather scientific decision points, e.g. the need to work on zero emission concepts and technology in order to achieve a given global climate target with a certain probability.

The set of all manipulated parameters or the set of all assumptions in tools plus qualitative assumptions that also may be implicitly mapped in the synthesis is one possible scenario. The basis for this is an earlier parameterization of the ATS. Since quantitative scenario development needs to be model-based 


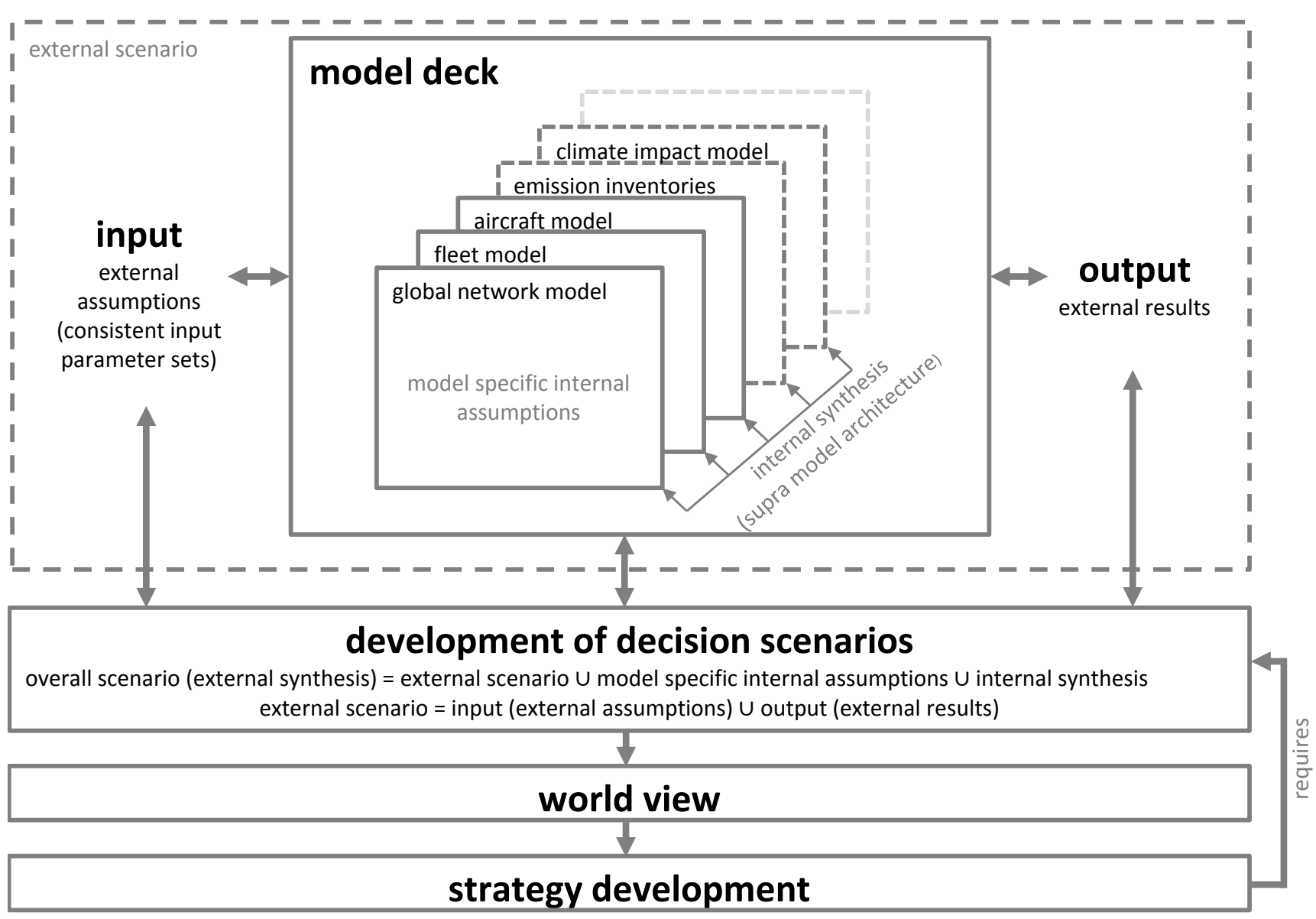

Figure 7: Scenario creation by deliberate model manipulation. A scenario is theoretically the set of all assumptions made - may they be internal or external, quantitative or qualitative, explicit or implicit. Strategies are developed based on a world view. Decision scenarios are built to question the world view of decision makers.

but at the same time cannot exclusively rely on numerical tools, the inherently quantitative scenario theory in combination with systems design was developed. This reasoning starts from the premise that in order to develop quantitative scenarios or to assess derivations of the ATS, the ATS needs to be "conceptually designed", which is reflected in a certain model deck and parameterization. The scenario is a way of organizing the smorgasbord of assumptions consistently in a storyline or timeline of events.

As aircraft design can be conducted putting together all the relevant information while understanding all the necessary trade-offs, the same should be done for the ATS as a whole which might serve as a basis to develop relevant scenarios.

\section{Scenario pull case - Global climate target and global fleet emission scenarios}

As an example of the scenario pull path as described in Section 3, explorative and normative scenarios have been developed in terms of climate change in [14]. Extent and urgency of action has been analyzed by contrasting emission scenarios of the global fleet with a global climate target of staying below $2{ }^{\circ} \mathrm{C}$ warming compared to pre-industrial levels. Only this evaluation of different scenarios and the decision on a desirable future can be a basis of requirements deduction for the future ATS. It needs to be evaluated by how much one scenario might differ from the other and why one might be more desirable. The hypothetical selection of one of the scenarios is 
related to a future requirement for the ATS and to elements of the ATS such as the aircraft. In the example of the $2^{\circ}$ target, this scenario analysis leads to the insight that the introduction of zero emission aircraft into the world fleet might be required at a certain point of time in the future, assuming continued traffic growth. For the development of useful decision scenarios it would not be necessary to ask whether the future will unfold like this, but to engage in the thought experiment to think societal problems and revolutionary concepts to their end. The need for the introduction of zero emission aircraft further implies that technologies and designs are found that can meet this requirement at a future point in time. These needed concepts are not confined to aircraft technology, but include all aspects of the ATS and even external aspects such as the characteristics of the future post-fossil energy system.

\section{Technology push case - Laminar flow technology or why not to start with scenarios}

As an example of the technology push path, the overall effects of natural laminar flow technology are being evaluated. Experiences with the assessment of laminar flow technologies show that in a technology-induced case, it is advisable to end with scenarios rather than to start with them. A scenario process is not likely to yield any valuable output at the beginning of a technology push case. During the systems analysis, e.g. for laminar flow technology, from technology level over aircraft design level to fleet level more and more assumptions have to be made. Static scenarios may help to cope with and to formalize the amount of assumptions. At the end of the process, dynamic external scenario parameters, e.g. the oil price development, can be included in the systems analysis in order to test sensitivities of go/no-go for natural laminar flow technology decisions under diverse external scenarios as conducted in [26] and [27].

\section{Conclusion}

The most surprising scenarios with the highest academic relevance for aeronautical research could be developed in manipulating external and internal assumptions likewise. Additionally, a scenario is defined by the internal synthesis, the mental model of the ATS itself, reflecting the assumption on how the ATS works and on how the main stakeholders interrelate. We showed that it is favorable not to treat scenarios as mere input for a tool chain, since internal assumptions forming a part of the scenario would be neglected that way. The Scenario Gaming approach may satisfy the need to work with numerical models and to be participatory at the same time. This is important because, as Schwartz puts it, "scenario making is intensely participatory, or it fails." [17] That means that the target to deduce requirements for the ATS of the future from divergent scenarios cannot be achieved successfully in a linear process or with a passive perception of the future. Requirements derivation or policy planning concerning the future ATS both need a normative and participatory approach to futurology, not a passive and descriptive one. The main advantage of the proposed framework over qualitative scenario technique is its two-way ability to develop scenarios from a technology push perspective and a scenario pull perspective quantitatively. The systematic level by level synthesis and iteration from technology to design to fleet operation and vice versa, enables a more realistic display of alternatives for action.

\section{References}

[1] Strohmayer, A. Szenariomethoden im Vorentwurf ziviler Transportflugzeuge, Verlag Dr. Hut, München, 2002.

[2] Phleps, P. Szenariobasierte Methode zur ökonomischen Lärm- und Emissionsbewertung zukünftiger Flugzeugkonzepte, Verlag Dr. Hut, München, 2011.

[3] Meussen M. and Becker, A. Intergration of a scenario method in Airbus' technology and project evaluation. 24th ICAS Conference, 2004.

[4] Meussen, M., Trunk, M., Henke, R. Long term aircraft project and technology evaluation under the 
uncertainty of future scenarios. 26th ICAS Conference, 2008.

[5] Eelman, S. An enhanced scenario approach assessing uncertainties in the realization of new aircraft and technologies. 25th ICAS Conference, 2006.

[6] Kahn, H and Wiener, A. The Year 2000: A Framework for Speculation on the Next Thirty-Three Years. Collier Macmillan, 1968.

[7] Ulf Pillkahn. Trends und Szenarien als Werkzeuge der Strategieentwicklung. Publics Corporate Publishing, Erlangen, 2007.

[8] Weiss, M. et al. Enhanced Assessment of the Air Transportation System. 11th AIAA Aviation Technology, Integration, and Operations (ATIO) Conference, Virginia Beach, 2011.

[9] Housing Industry Association of Australia. An Introduction to PESTLE Analysis. Small Business Information Series, http://hia.com.au/upload/hia/ documents/business information services/ sbis_guides/pestle_analysis.pdf, 2011.

[10] Gülden, K., Nolte, P., Wicke, K., and Stumpf, E. Utilizing scenario technique to derive and select robust requirements for a Concept of Operations in the Air Transportation System. 11th AIAA Aviation Technology, Integration, and Operations (ATIO) Conference, Virginia Beach, 2011.

[11] Stumpf, E., Langhans, S., Weiss, M., Sun, X. and Gollnick, V. A Methodology for Holistic Air Transport System Analysis. 11th AIAA Aviation Technology, Integration, and Operations (ATIO) Conference, Virginia Beach, 2011.

[12] Wack, P. Scenarios: Uncharted waters ahead. Harvard Business Review, Vol. 63, No. 5, pp 73-89, September 1985.

[13] Wack, P. Scenarios: shooting the rapids. Harvard Business Review, pp 2-14, November-December, 1985.

[14] Ghosh, R. and Gollnick, V. The $2^{\circ}$ target revisited: Climate-ecological assessment loop and conceptual foundation for air transportation system reengineering. Deutscher Luft- und Raumfahrt Kongress (DLRK), Stuttgart, Germany, 2013.

[15] Gausemaier, J., Plass, C., and Wenzelmannn, C.: Zukunftsorientierte Unternehmensgestaltung, Carl Hanser Verlag, München, 2009.

[16]Mietzner, D.. Strategische Vorausschau und Szenarioanalysen: Methodenevaluation und neue Ansätze. Gabler Verlag, 2009.

[17] Schwartz, P. The art of the long view. John Wiley and Sons, 1996.

[18] Quade, E. and Boucher, W. Systems Analysis and Policy Planning. Applications in Defense. American Elsevier Publishing Company, 1968.

[19] Kahn, H. and Mann, I. Ten Common Pitfalls. The Rand Corporation, 1957.

[20] Quade, E. Systems Analysis: A Tool for Choice. The Rand Corporation, USA, 1972.
[21]Kahn, H. and Mann, I. War Gaming. P1167,http://www.rand.org/content/dam/rand/pubs/pap ers/2006/P1167.pdf, 1957.

[22] Tsuchiya, S. Simulation/Gaming as an Essential Enabler of Organizational Change. Simulation \& Gaming, Vol. 29, No. 4, 400-408, December 1998.

[23] Joldersma, C. and Geurts, J.. Simulation/gaming for policy development and organizational change. Simulation and Gaming - Special issue on policy development and organizational change. Vol. 29, No. 4, pp 391-399, December 1998.

[24] Crookall, D. and Thorngate, W. Acting, Knowing, Learning, Simulating, Gaming. Simulation and Gaming, Vol. 40, No. 1, pp 8-26, February 2009.

[25] Bachmann, A., Lakemeier, J. and Moerland, E. An Integrated Laboratory for Collaborative Design in the Air Transportation System. Concurrent Engineering Approaches for Sustainable Product Development in a Multi-Disciplinary Environment, pp 1009-1020. Springer. 19th ISPE International Conference on Concurrent Engineering - CE2012, Trier, Germany, 2012.

[26] Wicke, K., Kruse, M., Linke, F., and Gollnick, V. Impact of insect contamination on operational and economic effectiveness of aircraft with natural flow technology. 29th Congress of the International Council of the Aeronautics Sciences (ICAS), St. Petersburg, Russia, 2014.

[27] Wicke, K., Kruse, M., and Linke, F. Mission and Economic Analysis of Aircraft with Natural Laminar Flow. 28th Congress of the International Council of the Aeronautical Sciences (ICAS 2014), Brisbane, Australia, 2012.

\section{Contact Author Email Address}

mailto:robin.ghosh@dlr.de

\section{Copyright Statement}

The authors confirm that they, and/or their company or organization, hold copyright on all of the original material included in this paper. The authors also confirm that they have obtained permission, from the copyright holder of any third party material included in this paper, to publish it as part of their paper. The authors confirm that they give permission, or have obtained permission from the copyright holder of this paper, for the publication and distribution of this paper as part of the ICAS 2014 proceedings or as individual off-prints from the proceedings. 